\title{
Hormonal evidence for induced ovulation in Monodelphis domestica
}

\author{
L. A. Hinds ${ }^{1}$, M. Reader ${ }^{2}$, S. Wernberg-Moller ${ }^{3}$ and N. R. Saunders ${ }^{2}$ \\ ${ }^{1}$ CSIRO Division of Wildlife and Ecology, PO Box 84, Lyneham, 2602, ACT, Australia; and \\ ${ }^{2}$ Clinical Neurological Sciences Group, South Academic Block, Southampton General Hospital, \\ Tremona Road, Southampton, UK; and ${ }^{3}$ Department of Physiology and Pharmacology, University \\ of Southampton, $U K$
}

\begin{abstract}
Summary. Breeding activity in the South American grey opossum, Monodelphis domestica, is stimulated by the presence of males. This study presents the first analysis of changes in concentrations of plasma progesterone during pregnant and nonpregnant cycles.

In Expt 1, females were paired with either intact or vasectomized males to stimulate a reproductive cycle, or were isolated from males. Within 4-8 days of pairing, females showed marked changes in the size of the urogenital opening (vulva), which were paralleled by similar changes in body weight $(11.4 \pm 2 \%$; mean \pm SEM; $n=13)$. There was a second increase and decrease in body weight $(21 \cdot 7 \pm 3 \cdot 3 \% ; n=13)$ in pregnant and nonpregnant cycles during the luteal phase of the cycle. Changes in concentrations of plasma progesterone, determined from samples collected three times a week, were similar in pregnant and nonpregnant cycles. Plasma progesterone concentration, which was increased for 15-18 days, showed two distinct peaks. The first peak, $3-4 \cdot 5 \mathrm{ng} \mathrm{ml}^{-1}$, occurred immediately after the decrease in vulval swelling, and the second peak (1-2 $\mathrm{ng} \mathrm{ml}^{-1}$ ) occurred 8-12 days later. Concentrations of plasma progesterone were low before parturition occurred. Females isolated from males for 35 days showed no changes in body weight or vulval swelling and concentrations of plasma progesterone remained undetectable.

In Expt 2, concentrations of luteinizing hormone ( $\mathrm{LH}$ ) in plasma were determined twice a day for the first few days after pairing. In three of five females an LH peak was detected coincident with the maximal swelling of the vulva.
\end{abstract}

Keywords: progesterone; pregnancy; oestrous cycle; luteinizing hormone; grey opossum; marsupial; induced ovulation

\section{Introduction}

In several eutherian species, the presence of a male, of male-related pheromones or of both (Whitten, 1966; Milligan, 1980) stimulates oestrus and ovulation in females (Whitten, 1956; Hughes, 1964; Carter et al., 1980). In most marsupials, ovulation occurs spontaneously (TyndaleBiscoe \& Renfree, 1987); the phenomenon of male-induced oestrus and ovulation in females has been reported in only two species, the South American grey, short-tailed opossum (Monodelphis domestica), a member of the most ancient marsupial family, the Didelphidae (Fadem et al., 1982; VandeBerg, 1983), and an Australian marsupial, a small ratkangaroo, the brushtail bettong, Bettongia penicillata, of the superfamily Macropodoidea (Smith, 1992; Hinds \& Smith, 1992). In several other marsupials, including the woolly opossum, Caluromys philander, although ovulation will occur spontaneously, the frequency and synchrony of oestrus can be enhanced by the presence of males (Perret \& Ben M'Barek, 1991). 
In $M$. domestica, stimulation of oestrus and ovulation is primarily influenced by social and olfactory cues (Fadem et al., 1982; VandeBerg, 1983; Fadem, 1985; Fadem \& Rayve, 1985; Fadem, $1986,1987)$. Unlike the woolly opossum, in which isolated females continue to undergo infrequent oestrous cycles (Perret \& Ben M'Barek, 1991), female $M$. domestica isolated from males for long periods reportedly become sterile (Maliniak \& Taft, 1981). However, breeding activity can be stimulated in $M$. domestica by the introduction of a male (Fadem et al., 1982), with sensory contact alone effectively inducing oestrus in $60 \%$ of females (VandeBerg, 1983; Fadem, 1985). Females are also stimulated when moved to cages previously occupied by intact males (Fadem, 1987; 1989). Nevertheless, it is not only the presence of a male, but also, more particularly, the introduction of a new male, that stimulates breeding (Fadem et al., 1982; VandeBerg, 1983; Fadem, 1985; Fadem \& Rayve, 1985). After the introduction of a new male, a female enters oestrus and most conceptions occur within 9 days; births occur 19-24 days after the initial pairing, indicating a gestation period of 14-15 days (Fadem, 1985; VandeBerg, 1983).

Fadem (1989) observed differences in oestradiol concentrations in plasma collected from females on two occasions, before and during pheromonal stimulation. However, sequential changes in any of the reproductive hormones during an oestrous cycle or pregnancy have not been described. The present study determined concentrations of progesterone in plasma from females paired with intact or vasectomized males and in females isolated from males. Consistent changes in vulval appearance and body weight were considered in relation to changes in concentrations of progesterone or LH in plasma.

\section{Materials and Methods}

\section{Animals}

The grey short-tailed opossum, $M$. domestica, is a South American didelphid marsupial from the Chaco, Cerrado and Caatinga regions of Brazil (Streilein, 1982). The adult female, which weighs approximately $70-120 \mathrm{~g}$, reproduces successfully under suitable laboratory conditions throughout the year (Fadem et al., 1982; VandeBerg, 1983). The colony of $M$. domestica at University of Southampton, UK, was established from eight breeding pairs kindly supplied by Mark Ferguson (University of Manchester, UK).

Most animals were held singly or in pairs for breeding in rat boxes made of polycarbonate or polypropylene and containing a deep layer of wood shavings. Generally, females were paired with males for 13 days after which they were separated and placed in plastic nesting boxes $(19 \times 11 \times 7 \mathrm{~cm})$ filled with newspaper strips and wood shavings. Animals were fed daily on a feline carnivore diet (Special Diet Services, Stepfield, Witham, Essex, UK) and pregnant animals were supplemented with Farex (Farley Health Products Ltd, Nottingham, UK). Water was freely available. The animal room was maintained at an ambient temperature of $27^{\circ} \mathrm{C}$ and a cycle of $14 \mathrm{~h}$ light: $10 \mathrm{~h}$ dark.

\section{Assessment of vulval swelling}

Previous observations of females paired with males indicated that there were significant changes in the size of the vulva/urogenital opening soon after pairing was established. To assess these changes objectively, the swelling of the vulva was scored once or twice a day and given a rating from 1 to 5 (1, small, pale, inactive vulva; 5 , enlarged, vascular vulva) (Fig. 1).

\section{Vasectomy}

Six male $M$. domestica were vasectomized using a procedure similar to that described for the eastern quoll, Dasyurus viverrinus (Hinds, 1989). Vasectomies were performed at least 2 weeks before the males were paired with any females used in this study.

\section{Collection of blood samples}

Animals were wrapped in an envelope of paper towelling, and placed in a purpose-built Perspex cage with the tail exposed. The lateral tail vein was punctured with a $26 \mathrm{~g}$ needle and blood (about $150-200 \mu \mathrm{l}$ ) collected into three or four $50 \mu \mathrm{l}$ heparinized capillary tubes. The samples were transferred to microcentrifuge tubes and held on ice until they 

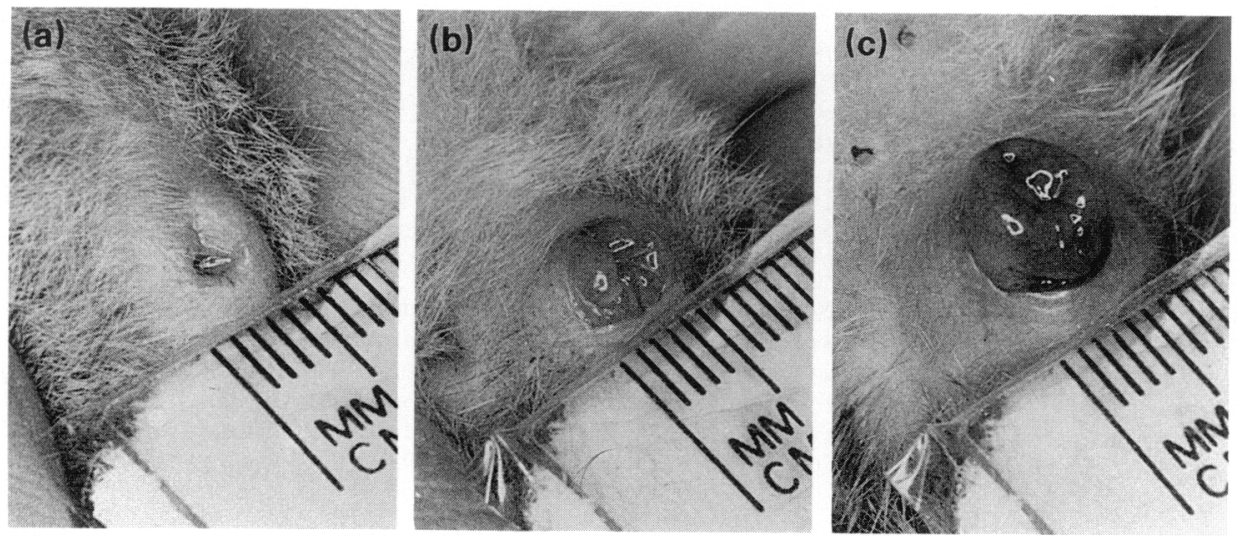

Fig. 1. Changes in the size and appearance of the everted urogenital opening (vulva) of a female Monodelphis domestica which was activated by the presence of a male. (a) Rating 1, vulva small, pale and inactive; (b) rating 3, vulva enlarging, more vascular; (c) rating 5 (maximum size), vulva enlarged, oedematous and well vascularized.

were centrifuged for $3 \mathrm{~min}$ at $3500 \mathrm{~g}$. The volume of plasma $(25-100 \mu \mathrm{l})$ was recorded, and the plasma was frozen and then freeze-dried. The lyophilized samples were sealed with parafilm, stored in a desiccator at room temperature and subsequently sent to Canberra for analysis.

\section{Hormone assays}

Progesterone. The radioimmunoassay originally described for the tammar wallaby, Macropus eugenii (Sernia et al., 1980), has been validated for $M$. domestica. The lyophilized samples $(25-100 \mu l)$ were reconstituted with double distilled water, made up to the same volume $(300 \mu \mathrm{l})$ with assay buffer $(0 \cdot 2$ mol sodium phosphate buffer 1 ', $\mathrm{pH} 7 \cdot 0$ in $0.9 \%$ saline plus $1.0 \%$ gelatin) and then extracted twice with 20 volumes of freshly redistilled $n$-hexane. Antiserum 9817, a gift from R. I. Cox (CSIRO Division of Animal Production, Sydney, Australia) was used at a final dilution of 1:224 000. Duplicate samples of plasma $(10-50 \mu \mathrm{l})$ were measured. The sensitivity of the assay was $10 \mathrm{pg}$ per tube or $0.25 \mathrm{ng} \mathrm{ml}$ ' plasma, and the hexane solvent blank and charcoal-treated (steroid-free) plasma blank always measured less than these limits. After extraction with freshly redistilled $n$-hexane the recovery of $\left[{ }^{3} \mathrm{H}\right]$ progesterone from $M$. domestica plasma was $98.2 \pm 0.6 \%$ (mean $\pm \mathrm{SD}, n=6$ ). The recovery of unlabelled progesterone added to charcoaltreated plasma was within $7 \%$ of that expected, while the intra- and interassay coefficients of variation were $7.5 \%$ $(n=12)$ and $9.5 \%(n=7)$, respectively. Serial dilutions $(5-100 \mu \mathrm{l})$ of two pools of plasma collected from females in late pregnancy were parallel to the progesterone standard curve. Pooled plasma $(25-150 \mu 1)$ collected from adult males showed no displacement of label in the assay system. The addition of charcoal-treated plasma $(250 \mu \mathrm{l})$ had no effect on maximum binding ( $60 \%$ in both cases), and standard curves prepared in charcoal-treated plasma or buffer could be superimposed.

Luteinizing hormone. The heterologous radioimmunoassay for determining plasma LH in the tammar wallaby (Sutherland et al., 1980) has been partially validated for $M$. domestica plasma, using antiserum GDN 15 (a gift from G. Niswender) and rat LH-I-6 as tracer and NIH-oLH-S-2l as standard. No pure M. domestica LH was available to test for parallelism with the ovine standard curve. However, serial dilutions of a pituitary homogenate were parallel to the standard curve and the recovery of ovine LH added to $M$. domestica plasma was within $10 \%$ of the expected value. The intra-assay coefficient of variation was $8 \cdot 2 \%(n=12)$.

\section{Experimental design}

Two experiments were performed to determine hormonal changes in females undergoing various reproductive cycles. In Expt 1, the changes in concentration of progesterone in plasma were determined in pregnant and nonpregnant females and in isolated females. In Expt 2, the presence and timing of the pre-ovulatory LH surge was determined. In both experiments, changes in body weight and in the appearance of the vulva were recorded. Initially, changes in the proportions of cell types in vaginal smears were to be used to determine oestrus, but the technique proved unsatisfactory because most smears contained too few cells for reliable diagnosis.

Experiment 1. Three groups of females $(50-90 \mathrm{~g})$ were established. Females of group I $(n=9)$ were paired with intact males and those of group $2(n=6)$ with vasectomized males to examine changes in concentration of progesterone in plasma in pregnant and nonpregnant cycles respectively. The day of pairing with a male was designated day 0 . 
Beginning on day 0 , body weights were recorded daily and for the next $8-12$ days changes in the size of the urogenital opening (vulval swelling) were assessed. Males were removed between days 13 and 20 . Approximately 8 days after the maximum swelling of the vulva was attained the females were palpated to assess the size of the uteri. The palpation was performed without knowledge of the expected reproductive state of the female. Blood samples were taken three times each week for analysis of progesterone in plasma.

Another group of five females (group 3) was isolated from all contact with males for 35 days to determine whether females underwent oestrous cycles in the absence of males. Vulval swelling was assessed daily, body weight changes were recorded on each weekday, and blood was sampled for progesterone determination three times a week. Animals were palpated as described for group 1 .

Experiment 2. The relationship between vulval swelling, body weight changes and the pre-ovulatory LH peak was determined by pairing females $(n=5)$ with intact males and blood samples were taken twice a day (at $08: 30 \mathrm{~h}$ and 17:00 h) beginning after the vulval rating reached $2-3$ and ending after the vulval rating decreased to $<4$; these samples were analysed for LH. Because the body size of $M$. domestica is small, no blood samples were taken for analysis of progesterone throughout the cycle, but animals were palpated 8 days after maximal vulval swelling and body weights were recorded for 25 days after pairing.

\section{Statistical analyses}

Paired $t$ tests were used to determine differences among various parameters for each reproductive state. The parameters compared were the intervals from pairing to the first rise in progesterone and to birth, and the concentrations of progesterone at different times during pregnant and nonpregnant cycles. All results are presented as means $\pm \mathrm{SEM}$.

Three criteria were used to assess stimulation by a male: (i) changes in the swelling of the vulva, (ii) changes in body weight and (iii) a subsequent increase in progesterone from $<0.5$ to $>1 \mathrm{ng} \mathrm{ml} \mathrm{'}$.

\section{Results}

\section{Changes in vulval size and body weight in the presence of males}

Before association with a male the urogenital opening/vulva was pale and small (Fig. la, rating 1-2). Within 2-5 days of pairing, there were distinct changes in all stimulated animals in the colour and degree of enlargement of the everted vulva (Fig. 1b, rating 3). The maximum size of the vulva was reached within 4-8 days in most animals (Fig. Ic, rating 5). During the next 2-3 days the size of the vulva decreased to rating $1-2$.

Parallel increases and decreases $(11.4 \pm 2 \% ; n=13)$ in body weight accompanied these changes in the vulva (Figs $2 \mathrm{~b}, \mathrm{~d}, 3 \mathrm{~b}$ and $5 \mathrm{a}-\mathrm{e}$ ). After the vulval score had fallen to rating $1-2$, there was a second increase in body weight $(21 \cdot 7 \pm 3 \cdot 3 \% ; n=13)$ which reflected the increase in the size of the uterus. In all females stimulated by the presence of a male, the uteri could be readily palpated 8 days after maximal vulval size (Figs $2 b, d$ and $3 b$ ). Unstimulated or isolated females showed no significant changes in body weight or size of the vulva, and the uteri remained small (Fig. 4).

\section{Experiment 1. Progesterone profiles in stimulated and unstimulated females}

Group 1. Eight of nine females paired with an intact male showed evidence of stimulated breeding activity, and five of these gave birth. The remaining female was not stimulated by the presence of a male and remained unstimulated when monitored for a further 35 days in the absence of a male. Progesterone concentrations in plasma showed a marked increase within two days of the maximal vulval score. The changes in the progesterone profile were very similar for all stimulated females (Figs 2a, c and 3a). The major difference between individuals both within and between groups was the day of the first increase above $1 \mathrm{ng} \mathrm{ml}^{-1}$. In group 1 this occurred on day 7 (range 5-13 days) (Fig. 2a) and in group 2 on day 9 (range 7-12 days) (Fig. 2c). Thereafter maximum concentrations and the pattern were similar. After the initial peak in progesterone $\left(3-4 \mathrm{ng} \mathrm{ml}^{-1}\right)$, a second peak of lower magnitude $\left(2 \mathrm{ng} \mathrm{ml}^{-1}\right)$ occurred 9-10 days later. Basal concentrations $\left(<0.5 \mathrm{ng} \mathrm{ml}^{-1}\right)$ were reached 14-15 days after the first increase above $1 \mathrm{ng} \mathrm{ml}^{-1}$. In females that produced a litter, progesterone concentrations in plasma were always less than $1 \mathrm{ng} \mathrm{ml}^{-1}$ on the day of or day after parturition (Figs 2a and 3a). In group 1, birth occurred in individual females 19, 20, 22, 23 and 

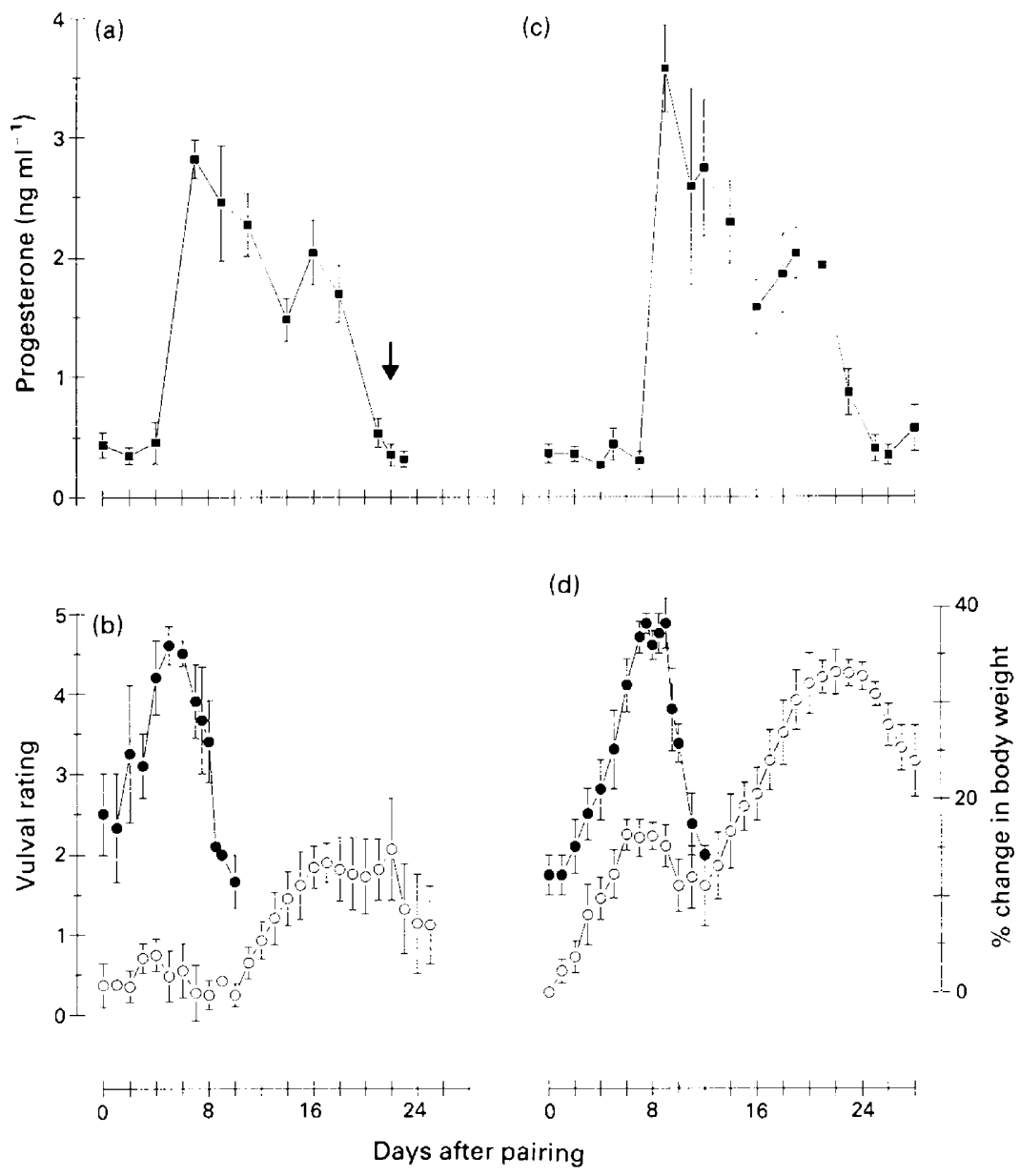

Fig. 2. Progesterone concentrations ( $\square$ ) in plasma (mean \pm SEM) for female Monodelphis domestica (a) during a pregnancy after pairing with an intact male $(n=5)$ and (c) during a nonpregnant cycle after pairing with a vasectomized male $(n=5)$. Values have been aligned on the mean day of the first increase in progesterone $>1 \mathrm{ng} \mathrm{ml}^{-1}$. Solid arrow indicates mean day of birth of litters. Corresponding changes in body weight $(O)$ and vulval swelling $(\boldsymbol{O})$, also aligned to first increase in concentration of progesterone in plasma, during (b) pregnancy and (d) the nonpregnant cycle, respectively.

27 days after pairing $(22.2 \pm 1.4$ days, mean \pm SEM), about 15 days after the first evidence of a decrease in the swelling of the vulva (Figs $2 a, b$ and 3 ).

One female in group 1 was retained with the same male although it was evident from palpation that she was not pregnant. She underwent a second cycle of stimulation as indicated by parallel changes in body weight and vulval size and increases in progesterone concentrations as in the first cycle. However she did not produce a litter. Another stimulated, but nonpregnant, female showed no subsequent changes in plasma progesterone after the intact male was removed from the cage.

Group 2. Five of the six females paired with a vasectomized male (group 2) showed evidence of stimulation. The remaining female showed an initial increase and decrease in both vulval size and body weight but no subsequent changes in body weight and no increases in plasma progesterone.

The profiles of progesterone concentration in plasma of activated females were similar to those of group 1 animals: the initial peak of progesterone was of similar magnitude and duration, and the 

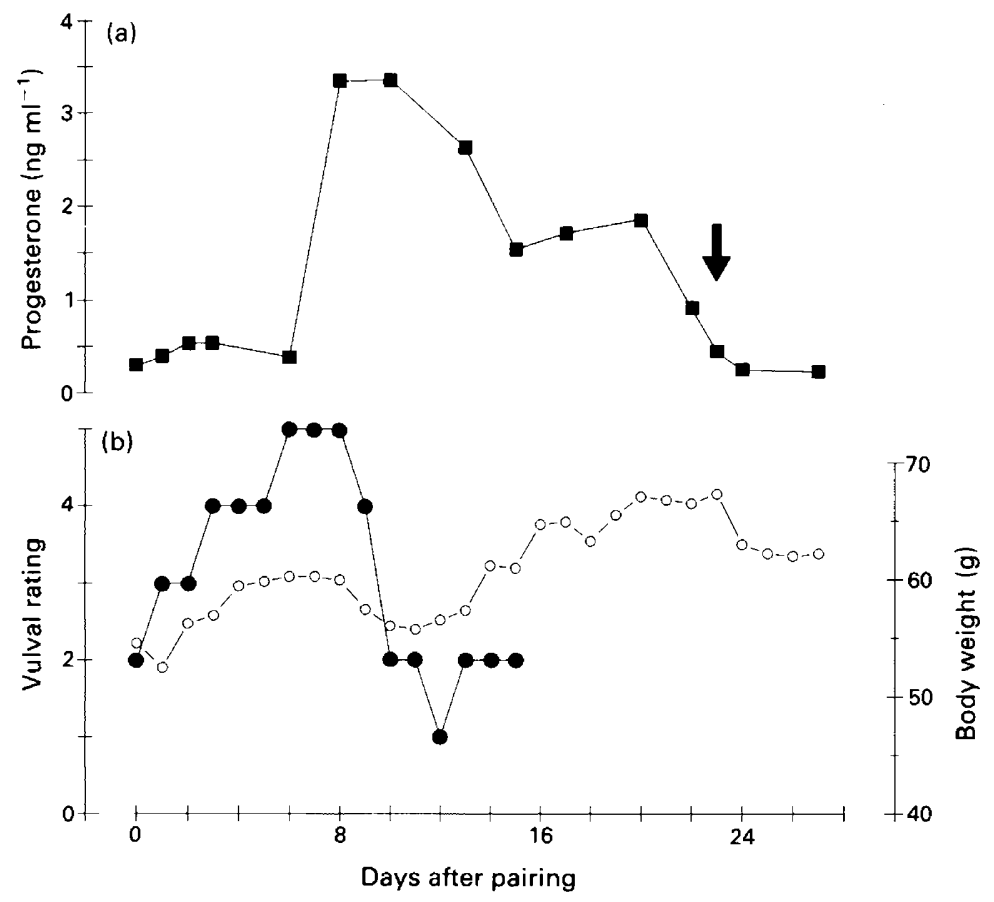

Fig. 3. Results for an individual female Monodelphis domestica during pregnancy. (a) Progesterone concentrations $\left(\mathrm{ng} \mathrm{ml}^{-1}\right)$ in plasma $(\boldsymbol{\square})$, and $(\mathrm{b})$ changes in body weight $(\mathrm{g})(\mathrm{O})$ and vulval rating (-). Solid arrow indicates day of birth of litter.

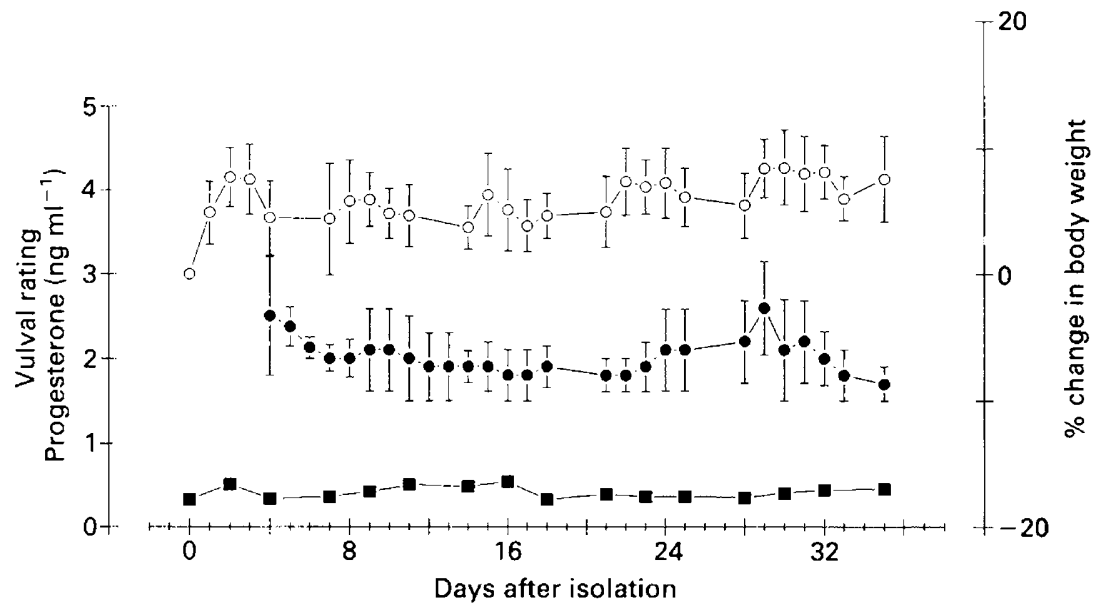

Fig. 4. Results for five female Monodelphis domestica isolated from males for 35 days. Progesterone concentrations $\left(\mathrm{ng} \mathrm{ml}^{-1}\right)$ in plasma (mean $\left.\pm \mathrm{SEM}\right)(\boldsymbol{\square})$, percentage change in body weight (mean \pm SEM) $(O)$ and changes in vulval rating (mean \pm SEM) $(O)$.

second peak of lesser magnitude was also present (Fig. 2c). Progesterone concentrations decreased to $<0.5 \mathrm{ng} \mathrm{ml}^{-1}$ about 15 days after the vulval rating began to decline. The increases in body weight (Fig. 2d) in the first few days after pairing and subsequently were greater than for group 1 

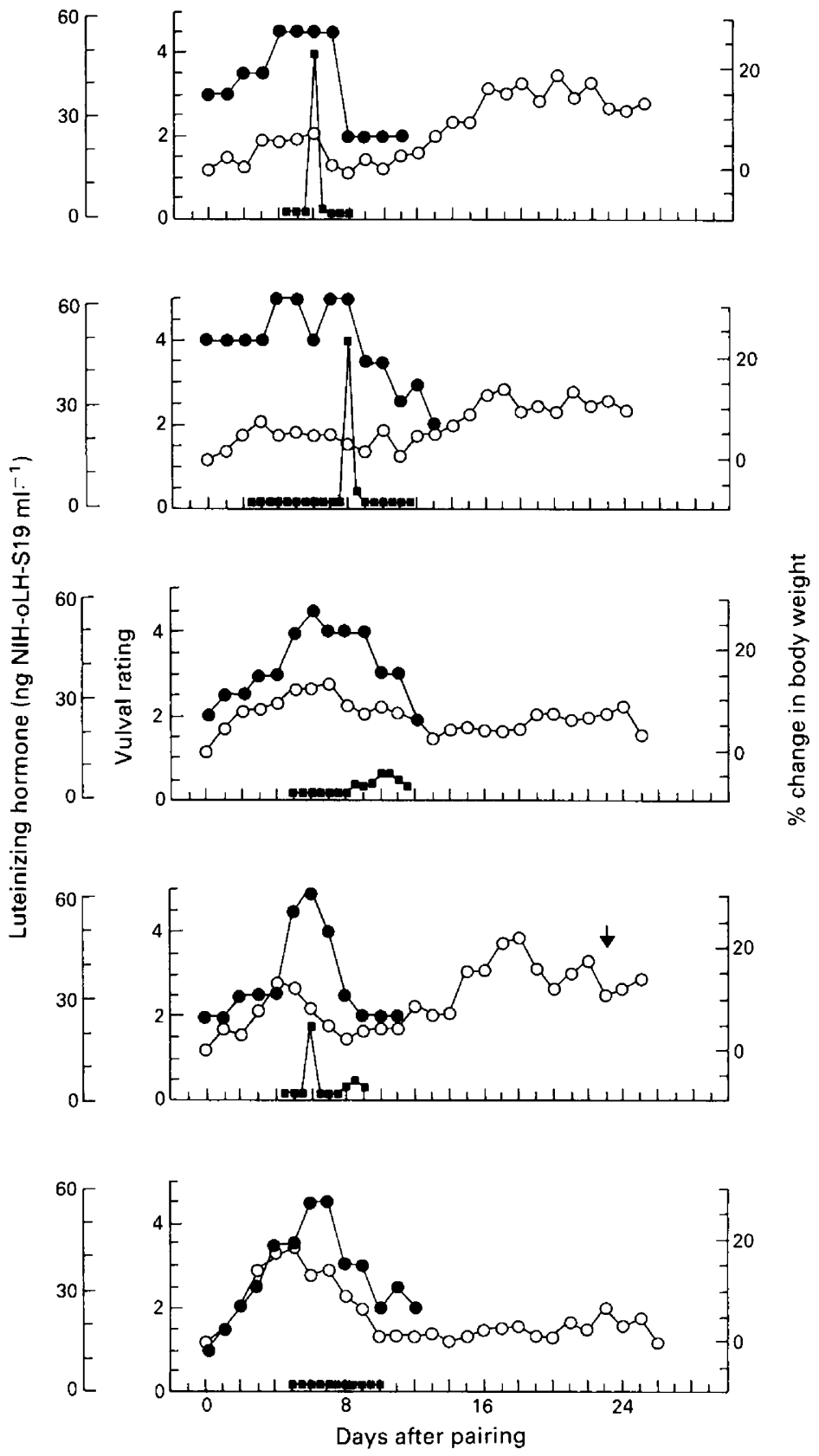

Fig. 5. Relationship between concentrations of luteinizing hormone (LH) in plasma ( $\mathbf{\square})$, changes in body weight $(O)$ and vulval ratings ( () in individual female Monodelphis domestica after pairing with intact males. Arrow indicates day of birth of litter.

females paired with intact males (Fig. 2b). The group 2 females were younger at the time of pairing and had not reached their mature body weight. Thus the changes in body weight associated with the cycle stimulated by the presence of a male occurred presumably along with a component of growth in these females. 
When females of groups 1 and 2 were palpated eight days after the swelling of the vulva had begun to decrease, pregnant and nonpregnant females could not be distinguished on the basis of uterine size. Five days later, the uteri of nonpregnant females were distinctly smaller than those of females that subsequently gave birth.

Group 3. None of the females isolated from males showed any evidence of stimulation during 35 days of observation. No changes in body weight, appearance of the vulva or increases in plasma progesterone were observed (Fig. 4). The absence of changes in the two females (one each from groups 1 and 2), which were kept in the presence of a male were identical to those of group 3.

\section{Experiment 2. Detection of the pre-ovulatory LH peak}

An LH peak was observed in three of five animals monitored twice daily after pairing with a male. The peak, ranging in magnitude from 8.4 to $>50 \mathrm{ng} \mathrm{ml}^{-1}$, occurred in two consecutive samples in two of three animals; LH concentrations remained low to undetectable at all other sampling times. All females showed evidence of stimulation in terms of body weight changes and in the swelling of the vulva (Fig. 5). Where the LH peak was detected it was coincident with the maximal swelling of the vulva and was followed by a second increase in body weight (Fig. 5a, b, d). In the absence of a detectable LH peak subsequent changes in body weight were not observed (Fig. $5 c, e)$. Only one female gave birth and this occurred 17 days after the occurrence of the LH peak (Fig. 5d).

\section{Discussion}

In previous studies of the reproductive physiology of Monodelphis domestica, activation of reproduction in females has been shown to follow the introduction of a female to a male (Fadem et al., 1982; VandeBerg, 1983; Fadem, 1985) or to his odours (Fadem, 1987). In this study, we provide the first hormonal evidence confirming that female $M$. domestica are induced ovulators. The introduction of either an intact or vasectomized male was equally effective in stimulating breeding activity in females. Activation was indicated by dramatic changes in the appearance of the vulva and corresponding changes in body weight, followed by a second rise in body weight and increases in plasma progesterone concentrations. By contrast, none of the females isolated from males for 35 days showed any of the above diagnostic changes.

Changes in the appearance and size of the vulva, in conjunction with parallel changes in body weight, provided an excellent indication of stimulation by a male. However, the appearance of these changes does not provide unequivocal evidence that ovulation will follow. For example in group 1, one female showed signs of stimulation 4-7 days after pairing, but no subsequent increase in body weight or changes in concentration of progesterone in plasma occurred. Similarly in Expt 2 , in two females there were changes in vulval size and body weight, but no LH peak was detected and no subsequent increase in body weight was observed. We conclude that ovulation did not occur in any of these females. Body weight changes in the pro-oestrous period are also seen in other marsupials, including Dasyuroides byrnei (Fletcher, 1989) and another South American marsupial, Marmosa robinsoni (Godfrey, 1975). In the latter species, during pro-oestrus the vaginal complex forms a sixth of the body weight of the animal, and the cloaca also becomes swollen and red. Female $M$. domestica in pro-oestrus have significantly higher concentrations of oestradiol in plasma than females that are not in oestrus (Fadem, 1989). Thus, the change in the size of the vulva in $M$. domestic $a$ and in other marsupials probably occurs in response to oestrogens produced by the growing follicles. Certainly there was a rapid decline in the vulval size coincident with or soon after the LH peak was detected, at which time, on conventional premise, the follicle would stop producing oestrogens and ovulation would occur (Harder et al., 1985).

The second increase in body weight in $M$. domestica, during the period when progesterone concentrations were high, was probably due to the increase in the size of the uteri which were easily 
detected by palpation at this stage. Until close to term the size of the uteri were similar in pregnant and nonpregnant females. Body weight changes during pregnancy and the oestrous cycle have also been reported for the kowari, D. byrnei (Fletcher, 1989).

In this study, as described in earlier studies (Fadem et al., 1982; Fadem, 1985, 1987), not all females became activated when associated with a male, and only about half of the pairings with intact males resulted in the birth of a litter. Fadem \& Rayve (1985) also report that isolated females undergo either short or long oestrous cycles ( 14 or 32 days), as determined from changes in cell types in vaginal smears. However, in the present study different criteria were used to assess reproductive activity and isolated females showed no evidence of ovulatory cycles nor were there increases in progesterone during the period of isolation. Because of the lack of progesterone measurements in the study of Fadem \& Rayve (1985), and our inability to obtain consistent vaginal smears, the results of the two studies cannot be reconciled. Clearly some females associated with males show vulval enlargement, but no subsequent progesterone increases which would indicate normal ovulation. Thus, although vaginal cornification may occur it is not accompanied by behavioural oestrus or ovulation, because pheromonal input was presumably inappropriate. Despite this interpretation, it is not clear why oestrous cycles of two lengths were observed in isolated females by Fadem \& Rayve (1985), unless, as in Marmosa robinsoni (Godfrey, 1975), the short cycles are anovular and the longer cycles are normal oestrous cycles.

The profile of concentration of progesterone in plasma was characterized by two peaks. The first peak occurred within 7-9 days after pairing and the second one, slightly lower in magnitude, 910 days later, about 16-19 days after initial pairing. This pattern is different from that reported for other species of this reproductive grouping (group 1, see Tyndale-Biscoe \& Renfree, 1987), the opossum (Didelphis virginiana), possum (Trichosurus vulpecula), kowari (Dasyuroides byrnei), eastern quoll (Dasyurus viverrinus) (see Hinds, 1990 for review) and woolly opossum (Caluromys philander) (Perret \& Ben M'Barek, 1991), in which the profile is clearly unimodal. The significance of the different pattern of progesterone concentration in $M$. domestica is unknown. Nevertheless, in $M$. domestica, as in the other group 1 species, pregnancy occupies the luteal phase only, and the profiles are similar in pregnant and nonpregnant females indicating that the lifespan of the corpora lutea appears to be unaffected by pregnancy. However, the results of the present study do suggest that the gestation period of $M$. domestica may be longer than 14-15 days (Fadem et al. 1982). The period of high progesterone concentration was never less than 15 days in any stimulated female, and birth always occurred after plasma progesterone concentrations had declined to less than $1 \mathrm{ng} \mathrm{ml}^{-1}$. Furthermore, in Expt 2, the only animal that gave birth did so 17 days after the LH peak was detected.

Induced ovulation is relatively common among eutherian species (Whitten, 1966; Milligan, 1980). However, the phenomenon has been described for only two marsupial species, the South American grey opossum, $M$. domestica (Fadem et al., 1982; VandeBergh, 1983) and the Australian potoroid, B. penicillata, of the Superfamily Macropodoidea (Smith, 1992; Hinds \& Smith, 1992). In the latter species induced ovulation had not been suspected because females were presumed to enter post-partum oestrus and ovulate spontaneously, as described for other species of kangaroo, wallaby and rat-kangaroo with a group 3 reproductive pattern (see Tyndale-Biscoe \& Renfree, 1987). Until recent studies compared concentrations of progesterone in plasma in $B$. penicillata females associated with, or isolated from, males in the post-partum period, it was not realised that the presence of males was critical for successful post-partum ovulation (Hinds \& Smith, 1992; Smith, 1992). Closer study of more marsupials may reveal other species in which spontaneous oestrus and ovulation does not occur in the absence of males.

In Southampton, expert assistance in animal handling and colony maintenance was provided by C. Bunce and C. Snart; in Canberra, assistance with hormone assays was provided by J. Wright. Part of this study was undertaken while L. A. Hinds was a participant in the Australian Academy 
of Science and Australian Academy of Technological Sciences Scientific and Technological Exchange Programme with the Royal Society.

\section{References}

Carter, C.S., Getz, L.L., Gavish, L., McDermott, J.L. \& Arnold, P. (1980) Male-related pheromones and the activation of female reproduction in the prairie vole (Microtus ochrogaster). Biology of Reproduction 23, 1038-1045.

Fadem, B.H. (1985) Evidence for the activation of female reproduction by males in a marsupial, the gray shorttailed opossum (Monodelphis domestica). Biology of Reproduction 33, 112-116.

Fadem, B.H. (1986) Chemical communication in gray short-tailed opossums (Monodelphis domestica) with comparisons to other marsupials and with reference to monotremes. In Chemical Signals in Vertebrates IV: Ecology, Evolution and Comparative Biology, pp. 587-607. Eds D. Duvall, D. Muller-Schwarze \& R. M. Silverstein. Plenum Publishing Corporation, NY.

Fadem, B.H. (1987) Activation of estrus by pheromones in a marsupial: stimulus control and endocrine factors. Biology of Reproduction 36, 328-332.

Fadem, B.H. (1989) The effects of pheromonal stimuli on estrus and peripheral plasma estradiol in female gray short-tailed opossums (Monodelphis domestica). Biology of Reproduction 41, 213-217.

Fadem, B.H. \& Rayve, R.S. (1985) Characteristics of the oestrous cycle and influence of social factors in grey short-tailed opossums (Monodelphis domestica). Journal of Reproduction and Fertility 73, 337-342.

Fadem, B.H., Trupin, G.L., Maliniak, E., VandeBerg, J.L. \& Hayssen, V. (1982) Care and breeding of the gray, short-tailed opossum (Monodelphis domestica). Laboratory Animal Science 32, 405-409.

Fletcher, T.P. (1989) Plasma progesterone and body weight in the pregnant and non-pregnant kowari, Dasyuroides byrnei (Marsupialia: Dasyuridae). Reproduction Fertility and Development 1, 65-74.

Godfrey, G.K. (1975) A study of oestrus and fecundity in a laboratory colony of Mouse opossums (Marmosa robinsoni). Journal of Zoology 175, 541-555.

Harder, J.D., Hinds, L.A., Horn, C.A. \& Tyndale-Biscoe, C.H. (1985) Effects of removal in late pregnancy of the corpus luteum, Graafian follicle or ovaries on plasma progesterone, oestradiol, LH, parturition and post-partum oestrus in the tammar Macropus eugenii. Journal of Reproduction and Fertility 75, 449-459.

Hinds, L.A. (1989) Plasma progesterone through pregnancy and the estrous cycle in the eastern quoll, Dasyurus viverrinus. General and Comparative Endocrinology 75, 110-117.

Hinds, L.A. (1990) Control of pregnancy, parturition and luteolysis in marsupials. Reproduction Fertility and Development 2, 535-552.
Hinds, L.A. \& Smith, M.J. (1992) Evidence from plasma progesterone concentrations for male-induced ovulation in the brush-tailed bettong, Bettongia penicillata. Journal of Reproduction and Fertility 95, 291-302.

Hughes, R. L. (1964) Effect of changing cages, introduction of the male and other procedures on the oestrous cycle of the rat. CSIRO Wildlife Research 9, 115-121.

Maliniak, E. \& Taft, L. (1981) Husbandry and breeding protocol for Monodelphis domestica. In Bulletin No. I Department of Zoology Research, pp. 1-4. National Zoological Park, Washington.

Milligan, S.R. (1980) Pheromones and rodent reproductive physiology. Symposia of Zoological Society of London 45, 251-275.

Perret, M. \& Ben M'Barek, S. (1991) Male influence on oestrous cycles in female woolly opossum (Caluromys philander). Journal of Reproduction and Fertility 91, 557-566.

Sernia, C., Hinds, L.A. \& Tyndale-Biscoe, C.H. (1980) Progesterone metabolism during embryonic diapause in the tammar wallaby, Macropus eugenii. Journal of Reproduction and Fertility 69, 139-147.

Smith, M.J. (1992) Evidence from the oestrous cycle for male-induced ovulation in Bettongia penicillata (Marsupialia). Journal of Reproduction and Fertility 95, 283-289.

Streilein, K.E. (1982) The ecology of small mammals in the semiarid Brazilian Caatinga. 111. Reproductive biology and population ecology. Annals of Carnegie Museum 51, 251-269.

Sutherland, R.L., Evans, S.M. \& Tyndale-Biscoe, C.H. (1980) Macropodid marsupial luteinising hormone: validation of assay procedures and changes in plasma levels during the oestrous cycle in the female tammar wallaby (Macropus eugenii). Journal of Endocrinology 86, $1-12$.

Tyndale-Biscoe, C.H.\& Renfree, M.B. (1987) Reproductive Physiology of Marsupials. Cambridge University Press, Cambridge, UK.

VandeBerg, J.L. (1983) The gray short-tailed opossum: a new laboratory animal. Institute of Laboratory Animals Research News 26, 9-12.

Whitten, W.K. (1956) Modification of the oestrous cycle of the mouse by external stimuli associated with the male. Journal of Endocrinology 13, 399-404.

Whitten, W.K. (1966) Pheromones and mammalian reproduction. Advances in Reproductive Physiology 1, 155-177.

Received 26 June 1991 